\title{
Super-Tonks-Girardeau regime in trapped one-dimensional dipolar gases
}

\author{
G.E. Astrakharchik \\ Departament de Física i Enginyeria Nuclear, Campus Nord B4-B5, \\ Universitat Politècnica de Catalunya, E-08034 Barcelona, Spain \\ Yu.E. Lozovik \\ Institute of Spectroscopy, 142190 Troitsk, Moscow region, Russia
}

(Dated: November 15, 2018)

\begin{abstract}
Possible signatures of a super-Tonks-Girardeau gas in bosonic systems of trapped quasi-onedimensional dipoles are discussed at zero temperature. We provide estimation of the frequency of the lowest compressional mode and compare it to analytical results derived using harmonic approach in the high density regime. We construct an exact mapping of the ground-state wave function of one-dimensional dipolar system of bosons, fermions and Bose-Fermi mixture and conclude that local properties and energy are the same at zero temperature. A question of to which extent the dipolar potential can be treated long- or short- range is discussed.
\end{abstract}

\section{INTRODUCTION}

Recent realization of chromium condensates [1] have brought much interest to unusual properties of dipolar condensates. Use of chromium have proved to be very advantageous as it has very large permanent dipolar moment of $\mu=6 \mu_{B}$. Other possible realizations of dipolar gases includ polar molecules [2, 3, 4] and laser-induced dipoles [5, 6]. Study of collective oscillations is a very sensitive tool for the investigation of the cold gas properties [7]. Dipolar forces compete with short-range $s$-wave scattering interaction. Although in the first experiments the $s$-wave interaction was giving dominant contribution to the energy, the effects of dipolar interactions have been detected in the shape of expanding cloud [8]. During the expansion of an initially trapped gas dipolar forces might lead to an anisotropic shape of an expanding cloud [9]. Use of Feshbach resonance technique have brought experiment to a new level. Indeed, by the means of Feshbach resonance technique it is possible to reduce $s$-wave isotropic contact interaction, such that the anisotropic magnetic dipole-dipole interaction between ${ }^{52} \mathrm{Cr}$ atoms becomes comparable in strength [10]. This induces large changes of the aspect ratio of the cloud, and, for strong dipolar interaction, the inversion of ellipticity during expansion can even be suppressed [10]. Chromium atoms have have as well fermionic ${ }^{53} \mathrm{Cr}$ isotope and Bose - Fermi cold mixtures have been recently realized [11].

Furthermore, dipolar interactions are very interesting as they contain both repulsive and attractive parts. Having attractive interactions between bosonic particles at temperatures so low that condensate is formed might lead to instability and a violent collapse process $12,13,14$. Collapse of large dipolar condensates in traps have been studied using non-local Gross-Pitaevskii 15, 16, 17, 18] theory and more precise Diffusion Monte Carlo (DMC) 17] approach.

One-dimensional (1D) cold systems have received great attention in the last years [19, 20, 21]. Role of quantum fluctuations is increased in reduced dimensionality leading to sometimes very different behavior. For example, a peculiarity of a one-dimensional world is an absence of a true Bose condensate in 1D homogeneous systems even at zero temperature [22]. There is a certain trend to study low-dimensional systems in the last years.

One of the most precise techniques, which can be used for testing the equation of state, is the measurement of the frequency of the "breathing" mode produced by a sudden change of the frequency of the harmonic trapping. We note that differences of several percent can be resolved in present high-precision experiments with cold gases (see, for example, Ref. [23]).

In this paper we address properties of a quasi-one-dimensional dipolar system at zero temperature. We provide an explicit mapping of the fermionic ground-state wave function and a wave function of Bose-Fermi mixture to bosonic wave function in one-dimension. Due to this mapping energy of dipolar systems containing fermions is predicted using results previously obtained for bosons. We calculate frequency of the lowest breathing mode in the trap and compare it to analytic predictions obtained in the high density limit. Measurement of the frequencies of collective oscillations can provide a signature of a super-Tonks-Girardeau (STG) regime 24, 25] described by very strong correlations (stronger than in the Tonks-Girardeau gas [26] in which the coupling constant is infinitely large). It is very difficult to reach STG regime in systems with short-range interactions, while its realization is feasible in dipolar systems. We address problem of superfluidity in one-dimensional systems and a question up to which extent dipolar interaction is shortor long- range. 


\section{MODEL}

A system of $N$ dipoles in one-dimensional geometry is described by the following model Hamiltonian:

$$
\hat{H}=-\frac{\hbar^{2}}{2 M} \sum_{i=1}^{N} \frac{\partial^{2}}{\partial z_{i}^{2}}+\frac{1}{2} \sum_{i=1}^{N} M \omega_{z}^{2} z_{i}^{2}+\frac{C_{d d}}{4 \pi} \sum_{i<j} \frac{1}{\left|z_{i}-z_{j}\right|^{3}}
$$

Here we assume that all dipoles are polarized and are oriented perpendicularly to the one-dimensional line. This stabilizes the system (as only repulsive part of dipolar interactions is relevant) and avoids collapses due to attraction. Expression for the coupling constant $C_{d d}$ depends on the nature of the dipolar interaction. For example, following realizations are possible:

1) Cold bosonic atoms, with induced or static dipole momenta, in a transverse trap so tight that excitations of the levels of the transverse confinement are not possible and the system is dynamically one-dimensional. The longitudinal confinement is described by the frequency $\omega_{z}$ of the harmonic trapping potential. The dipoles themselves can be either induced or permanent. In the case of dipoles induced by an electric field $E$ the coupling has the form $C_{d d}=E^{2} \alpha^{2}$, where $\alpha$ is the static polarizability. For permanent magnetic dipoles aligned by an external magnetic field one has $C_{d d}=m^{2}$, where $m$ is the magnetic dipole moment. We suppose that $s$-wave scattering length is tuned to zero by applying Feshbach resonance and only dipolar forces are relevant.

2) Spatially indirect excitons in two coupled quantum wires. A quantum wire is a semiconductor nanostructure where an electron or a hole is allowed to move only in one direction and excitations of the transverse quantization levels are negligible. In two parallel quantum wires, one containing only holes, and the other only electrons, holes and electrons couple forming indirect excitons. If such a system is dilute enough, it constitutes a $1 D$ set of dipoles. In this case $C_{d d}=e^{2} d^{2} / \varepsilon$, where $e$ is an electron's charge, $\varepsilon$ is the dielectric constant of the semiconductor, and $d$ is the distance between the centers of the quantum wires. This system is $1 \mathrm{D}$ counterpart of $2 \mathrm{D}$ indirect exciton system in coupled quantum wells.

\section{SUPER-TONKS-GIRARDEAU REGIME}

Properties of a homogeneous system $\left(\omega_{z}=0\right)$ have been studied numerically in [27] by means of Diffusion Monte Carlo method. The equation of state and correlation functions have been calculated as a function of the guiding parameter $n r_{0}$, where $r_{0}=M C_{d d} /\left(4 \pi \hbar^{2}\right)$ is a characteristic length, $n$ being the linear density. It has been found that the system is extremely correlated and shows crystal-like properties as the strength of dipolar interactions $n r_{0}$ is increased. This behavior is very different from the one of systems with short-range potentials, like the ones recently realized experimentally[28, 29]. Indeed, one-dimensional systems with $\delta$-interaction, $V(z)=-2 \hbar^{2} / m a_{1 D} \delta(z),($ LiebLiniger model[30]) have a completely different behavior of correlation functions 31, 32] which in this case is much closer to the one of a weakly interacting Bose gas. For example a typical shape of the static structure factor $S(k)$ is a smooth function which goes from zero for $k=0$ to asymptotic constant value $S(k)=1$ for large $|k|$. In the most strongly interacting limit of Lieb-Liniger system $-2 \hbar^{2} / m a_{1 D} \rightarrow+\infty$ (Tonks-Girardeau regime[26]) the static structure factor is equal to the one of an ideal Fermi gas and has a discontinuity in the derivative at momentum $|k|=2 k_{F}=2 \pi n$. Even stronger correlations might be achieved in a system with short-ranged potential by quickly crossing the confined induced resonance [33] as proposed in [24]. In this resulting "super-Tonks-Girardeu" regime bosonic atoms will interact with attractive potential $-2 \hbar^{2} / m a_{1 D} \rightarrow-\infty$. The true ground state in this regime is a soliton-like state with large and negative energy [34] ("attractive collapse" of the system). Instead, if the sweep across resonance is fast and does not significantly perturb position of the particles, the system will still remain in a gas-like state (which is metastable). As shown in Ref. [24], this state is dynamically stable if the gas parameter $n a_{1 D}$ is relatively small. The static structure factor in super-Tonks-Girardeau regime has a peak at $|k|=2 k_{F}$ and height of the peak increases for larger values of the gas parameter $n a_{1 D}$. The super-Tonks-Girardeau regime also exists in a number one-dimensional system (bosonic and fermionic) with infinite strength potentials, namely hardrods [35] and Calogero-Sutherland model[36]. So far this regime have never been observed experimentally with the best candidate for its observation being a system of dipoles. An experimental signature of STG regime in a trapped system is a frequency of lowest compressional mode $\Omega_{z}$ larger than $2 \omega_{z}[24]$. In the Section [V] we calculate explicitly the dependence of $\Omega_{z}$ on parameters of a trap.

Super-Tonks-Girardeau regime is expected to have quite unusual properties. It has been shown 37, 38, 39] for a Lieb-Liniger model that the dynamic form factor has a power low in the point where the excitation spectrum touches zero $S\left(\omega, 2 k_{F}\right) \propto \omega^{\eta-2}, \omega \rightarrow 0$, where $\eta=2 \hbar k_{F} / m c$. In the regime of repulsive $\delta$-interaction $\eta>2$ and $S\left(0,2 k_{F}\right)=0$. For the marginal case of Tonks-Girardeau regime $S\left(\omega, 2 k_{F}\right)=$ const. Instead for attractive $\delta$-interaction $(i . e$. in the Super-Tonks-Girardeau regime) this expression predicts a weak (power law) divergence in the dynamic form factor 


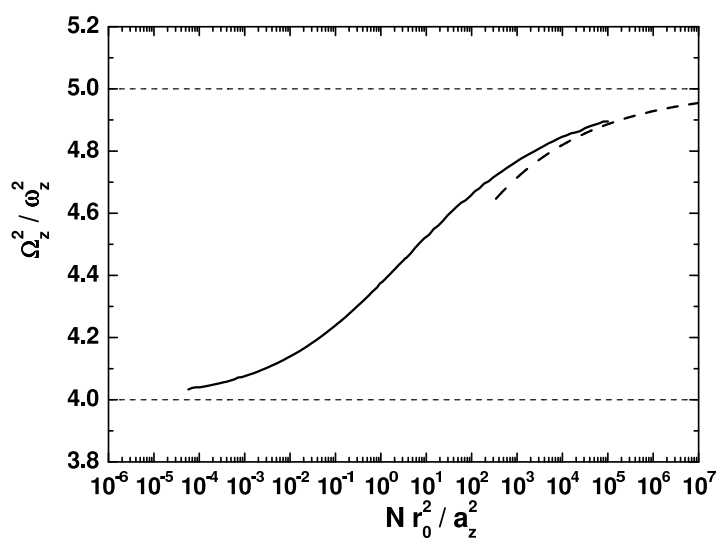

FIG. 1: Square of the lowest breathing mode frequency, $\Omega_{z}^{2}$, as a function of the coupling strength $N r_{0}^{2} / a_{z}^{2}$. The solid line: using equation of state of one-dimensional dipols (data is taken from [49], Fig. 6.5), dashed line: high density expansion, Eq. (33).

$S\left(\omega, 2 k_{F}\right) \rightarrow \infty, \omega \rightarrow 0$. For additional information on singularities in the dynamic structure factor for different values of momenta see Ref. [40, 41]

The problem of superfluidity has peculiarities in one-dimensional system. While different ways to define superfluid part in three- and two- dimensional systems are consistent, this is not the case in a one-dimensional world. Indeed, its calculation as a response of a liquid to sample boundary motion (winding-number method [42]) for the highly correlated states described by translationary invariant gas-like wave function (for example, exact wave function for the TonksGirardeau [26], Calogero-Sutherland [43, 44], hard-rods [26] systems; and DMC evaluation for Lieb-Liniger 31], dipolar [27] systems) would provide the result that such systems are completely superfluid. This argument would apply even to the TG regime, where bosonic system has many similar properties to an ideal Fermi gas. At the same time, one should keep in mind that exposure of an one-dimensional ideal Fermi gas to a tiny perturbation will change the ground-state wave function in a dramatic way: the overlap of the new wave function and the old is essentially zero. This effect known as orthogonality catastrophe (see, for example, textbook [45]) shows that in one-dimensional system stability to external perturbations have to be carefully checked. Contrary to winding number approach, Landau argument would lead to exactly opposite result classifying systems as completely normal. Indeed, in one-dimensional Luttinger liquids the excitation spectrum always touches zero at finite value of momentum, $|k|=2 k_{F}$ (see, for example, Ref. [39]; for Lieb-Liniger model the "type II" excitation[46] that touches zero can be identified as a dark soliton[47]). Another way to calculate superfluid density by the response to transverse probe (transverse current-current response) is not applicable within one-dimensional description as no transverse direction is included in the model. Contradictions in the results arise from different definitions of superfluid part and reflect the non-standard nature of the system. Probably the most natural and appropriate way to test the superfluidity in $1 \mathrm{D}$ world is done by dragging a small impurity (perturbation) through the system and seeing if this leads to energy dissipation. The force $F_{V}$, experienced by the system, depends on the interaction parameter $\eta$ as $F_{V} \propto V^{\eta-1}$ [38] where $V$ is velocity with which a small $\delta$-perturbation moves through the system. Thus in the mean-field limit $\eta \rightarrow \infty$ the force is vanishing and from a practical point of view system behaves analogously to a superfluid. On the contrary, in the Tonks-Girardeau limit $F_{V} \propto V$ and system behaves, from the point of view of friction, as a normal system, where the drag force is proportional to the velocity. In between there is a smooth crossover. The dipolar one-dimensional systems are expected to behave as normal ones.

Although the Bose-Einstein condensation is absent in a one-dimensional system even at zero temperature, its reminiscence still can be observed in divergence of the momentum distribution for $k=0$. This divergence is present in a homogeneous system of bosons in the Tonks-Girardeau regime. From the Luttinger liquid theory it is possible to show that deep in super-Tonks-Girardeau regime this divergence will be removed. This happens for $\eta=1$ (similarly to Calogero-Sutherland [36] and Hard-Rod [35] systems) or in terms of Luttinger parameter $K=1 / 2$. The Luttinger parameter can be extracted from the equation of state or from the phononic part of the static structure factor and is explicitly given in Ref. [48]. 


\section{FREQUENCIES OF COLLECTIVE OSCILLATIONS}

The equation of state obtained in [27] can be used to predict properties of a trapped one-dimensional gas within local density approximation (LDA) (see, for example, [50]) by assuming that the chemical potential in a trap is a sum of local chemical potential, taken to be the same as in a homogeneous system $\mu_{h o m}(n)$, and the external harmonic potential: $\mu=\mu_{\text {hom }}(n(z))+(1 / 2) M \omega_{z}^{2} z^{2}$. Within LDA number of particles $N$ and oscillator length $a_{z}=\sqrt{\hbar / M \omega_{z}}$ come in a single combination, $N r_{0}^{2} / a_{z}^{2}$. The LDA is expected to describe correctly properties of a gas in the trap if size of the cloud is large compared to $a_{z}$. From the knowledge of the density profile $n(z)$ one can obtain the mean square radius of the cloud $\left\langle z^{2}\right\rangle=\int_{-R}^{R} n(z) z^{2} d z / N$ and thus, making use of the result [51] $\Omega_{z}^{2}=-2\left\langle z^{2}\right\rangle /\left(d\left\langle z^{2}\right\rangle / d \omega_{z}^{2}\right)$ to calculate the frequency $\Omega_{z}^{2}$ of the lowest breathing mode.

Fig. 1 shows the square of the lowest breathing mode frequency $\Omega_{z}^{2}$ as a function of the coupling strength $N r_{0}^{2} / a_{z}^{2}$. In the TG regime, $N r_{0}^{2} / a_{z}^{2} \ll 1$, the frequency reaches a typical result of an ideal Fermi gas $\Omega_{z}=2 \omega_{z}$. The observation of a breathing mode with a frequency larger than $2 \omega_{z}$ would be a clear signature of the super-Tonks-Girardeau regime.

Attempt to calculate analytically the equation of state in the regime $n r_{0} \ll 1$ in terms of the $s$-wave scattering length and to obtain expansion for the frequencies of collective oscillations encounters problems as will be explained in Section VI. Instead some analytical results can be obtained in the regime of high densities $n r_{0} \gg 1$. In this regime the dipolar system has crystal-like properties 27]. At the same time the difference in the energy between gas-like and crystal-like descriptions is extremely small. This justifies an attempt to derive in a perturbative way the equation of state of a crystal and use it for an approximate description of the gas-like phase at the same density. We use classical crystal harmonic approach to calculate equation of state in the perturbative manner. Details of the calculation are provided in Appendix A The result for the equation of state is

$$
\frac{E}{N}=\zeta(3) \frac{\hbar^{2}}{M r_{0}^{2}}\left(n r_{0}\right)^{3}+C \frac{\hbar^{2}}{M r_{0}^{2}}\left(n r_{0}\right)^{5 / 2}+\ldots, n r_{0} \gg 1
$$

with $C=2.26 \ldots$. Application of the LDA to "perturbative" equations of state has been studied in details in [50]. Density profile, total and release energy are easily obtained by using expansion (2) in the high density $N r_{0}^{2} / a_{z}^{2} \gg 1$ regime. Here we report the frequency of the lowest compressional mode:

$$
\frac{\Omega_{z}^{2}}{\omega_{z}^{2}}=5-\frac{1.1358 \ldots}{\left(N r_{0}^{2} / a_{z}^{2}\right)^{1 / 5}}+\ldots
$$

The obtained dependence is shown in Fig. 1 as a dashed line and provides good description for large densities.

\section{BOSE-FERMI MAPPING FOR THE GROUND-STATE WAVE FUNCTION}

In the previous paper [27] we have calculated zero-temperature equation of state for the homogeneous Hamiltonian (11) with $\omega_{z}=0$ for bosonic particles and have sampled correlation functions over the ground-state wave function $\Psi_{B}\left(z_{1}, \ldots, z_{N}\right)$. As in experiments dipoles can be not only bosons, but as well fermions, or even a mixture of bosons and fermions we note that the equation of state we have obtained is applicable also to systems containing fermions. In order to prove that we construct an exact mapping of the wave function of bosonic dipoles to a wave function of fermionic wave function by analogy to what Marvin Girardeau did in his classical work[26]. For simplicity we start with a system of same-spin fermions. Such a system is described by the Hamiltonian (11). The main difference from the bosonic case is that fermionic wave function must be antisymmetric with the respect of exchange of any two particles. This can be done as

$$
\Psi_{F}\left(z_{1}, \ldots, z_{N}\right)=\prod_{i<j} \operatorname{sign}\left(z_{i}-z_{j}\right) \Psi_{B}\left(z_{1}, \ldots, z_{N}\right) .
$$

It is easy to check that the symmetry of wave function (4) is correct. Furthermore, due to Pauli exclusion principle, two Fermions are not permitted to stay in the same place $\Psi_{F}\left(z_{1}, \ldots, z_{N}\right)=0$ if $z_{i}=z_{j}$. This is already satisfied in the construction of bosonic wave function $\Psi_{B}\left(z_{1}, \ldots, z_{N}\right)$ due to divergence of $1 /|z|^{3}$ interaction for small $z$. It means that performed Diffusion Monte Carlo calculation for bosons is equivalent to Fixed-Node Diffusion Monte Carlo calculation for fermions with exactly known nodal structure as far as energy and local quantities (pair correlation function, static structure factor, etc.) are concerned. The trick (4) has been successfully used for study Fermionic Calogero-Sutherland model in Ref. [36]. 
Another system which will have similar zero-temperature equation of state is a system of two-component fermions, where the mass $M_{\sigma}$ of different spin atoms $\sigma=\uparrow, \downarrow$ is the same. Hamiltonian of such a system is given by

$$
\hat{H}=-\frac{\hbar^{2}}{2 M} \sum_{\sigma ; i=1}^{N^{\sigma}} \frac{\partial^{2}}{\partial z_{i, \sigma}^{2}}+\frac{1}{2} M \omega_{z}^{2} \sum_{\sigma ; i=1}^{N^{\sigma}} z_{i, \sigma}^{2}+\frac{C_{d d}}{8 \pi}\left(\sum_{i \neq j}^{N^{\uparrow}} \frac{1}{\left|z_{i}^{\uparrow}-z_{j}^{\uparrow}\right|^{3}}+\sum_{i \neq j}^{N^{\downarrow}} \frac{1}{\left|z_{i}^{\downarrow}-z_{j}^{\downarrow}\right|^{3}}+\sum_{i, j} \frac{1}{\left|z_{i}^{\uparrow}-z_{j}^{\downarrow}\right|^{3}}\right)
$$

Ground state wave function of system with totally $N=N^{\uparrow}+N^{\downarrow}$ atoms is than mapped onto a system of $N$ bosons with a Hamiltonian (11) as

$$
\Psi_{F}\left(z_{1}^{\uparrow}, \ldots, z_{N^{\uparrow}}^{\uparrow}, z_{1}^{\downarrow}, \ldots, z_{N \downarrow}^{\downarrow}\right)=\prod_{i<j}^{N^{\uparrow}} \operatorname{sign}\left(z_{i}^{\uparrow}-z_{j}^{\uparrow}\right) \prod_{i<j}^{N^{\downarrow}} \operatorname{sign}\left(z_{i}^{\downarrow}-z_{j}^{\downarrow}\right) \Psi_{B}\left(z_{1}^{\uparrow}, \ldots, z_{N}^{\uparrow}, z_{1}^{\downarrow}, \ldots, z_{N}^{\downarrow}\right) .
$$

Thus we conclude that a system one-dimensional Fermionic dipoles has the same ground-state equation of state as a system of bosonic dipoles. Consequently the LDA density profile in a trap is the same. This means that the frequencies of the lowest breathing mode for fermions follow the dependence shown in Fig. 11with $N$ being total number of dipoles. The limit $N r_{0}^{2} / a_{z}^{2} \rightarrow 0$ corresponds to ideal fermions and ideal fermions has the spherical breathing mode $\Omega=2 \omega_{h o}$ in any dimension (see, for example, [50]). For a finite value of $N r_{0}^{2} / a_{z}^{2}$ the frequency is increased due to repulsive interactions. Similar effects have been predicted in Ref. [52] using Bethe-ansatz theory for systems of two-component fermions with $\delta$-pseudopotential attractive [53, 54] and repulsive [55] interaction between atoms of different spin. While homogeneous system of bosons collapses if the interaction is attractive, this is not the case for fermions, where Pauli principle stabilizes the system. Adding weak attraction between atoms leads to softening the breathing mode $\Omega_{z}<2 \omega_{z}[52]$.

The Bethe ansatz method permits to find ground-state energy and, thus, study collective oscillations within LDA in a mixture of one-dimensional bosons and fermions with $\delta$-pseudopotential repulsive interactions and arbitrary bosonsfermions density ratio [56]. Such a mixture was found to be always stable against demixing [56]. Ground-state wave function of a mixture of one-dimensional bosons and fermions can be obtained with the same reasoning as for (4) and (6), thus leading to the same frequency of oscillations as in Fig. 1. Such kind of mapping turns out to be quite general. Indeed, it works for one-dimensional systems where one-dimensional interaction potential diverges when two particles meet and shows that energy, local quantities (pair-correlation function, three-particle correlation function, etc.) are the same for bosons and fermions at zero temperature.

\section{ARE DIPOLAR INTERACTIONS LONG-RANGE?}

It is common to oppose long-range dipolar interactions to short-range interactions described by $s$-wave scattering length. But are dipolar interactions really long range? Or to which extend are they long-range? There are at least two ways to approach this question.

The first way is to classify potential $V_{i n t}(r)$ as long- or short- range depending if the chemical potential is extensive on intensive quantity. If the homogeneous properties of a large system can be defined by density $n$ only (in appropriate units), $\mu=\mu(n)$, the potential is short-range. If instead number of particles $N$ have to be explicitly specified, $\mu=\mu(N, n)$, due to strong (diverging) dependence on $N$, the potential is of a long-range. This can be immediately checked by testing the convergence of the potential energy at large distances:

$$
I=\int_{L_{m i n}}^{\infty} V_{i n t}(r) r^{D-1} d r
$$

where $L_{\min }$ is some cut-off length and $D$ is dimensionality. Following this definition potential is short-range if it decays at large distances faster than $r^{-D}$ in $D$ dimensions (see, for example, [57]). From this point of view, $1 /|r|^{3}$ potential is long-range in $3 D$, while it is short-range in $1 D$ and $2 D$.

Alternatively, short-range potentials can be defined as potentials that can be described by the asymptotic phase shift. This means that a short-range potential of range $R$ can be approximated at large distances $r \gg R$ by a free-wave with an appropriate phase shift, or, being the same, the $s$-wave scattering length $a$. At sufficiently small densities $n a^{D} \rightarrow 0$, the only relevant length is $a$ and properties (for example energy) can be expressed in terms of the gas parameter $n a^{D}$. We use definition of the $s$-wave scattering length as a position of the node of analytic continuation of the scattering solution from distances larger than the range of the potential in the zero-energy scattering limit. This definition works well in three-dimensional systems, but also it is applicable to low-dimensional systems. 
It is possible to solve the two-body scattering problem for $1 /|r|^{3}$ potential at zero energy and find the scattering solution $f(r)$. One needs to look for a regular solution $(f(0)=0)$ of the following differential equation:

$$
-\frac{\partial^{2} f(r)}{\partial r^{2}}-\frac{D-1}{r} \frac{\partial f(r)}{\partial r}+\frac{f(r)}{r^{3}}=0
$$

where length is expressed in units of $r_{0}$ and energy in $\hbar^{2} / M r_{0}^{2}$. Solutions of (8) can be written explicitly $f^{3 D}(r) \propto$ $r^{1 / 2} K_{1}\left(2 r^{-1 / 2}\right), f^{2 D}(r) \propto K_{0}\left(2 r^{-1 / 2}\right), f^{1 D}(r) \propto r^{-1 / 2} K_{1}\left(2 r^{-1 / 2}\right)$, where $K_{n}(r)$ denotes the modified Bessel function of the second kind. In order to find the $s$-wave scattering length one has to expand $f(r)$ far from the range of the potential $(r \rightarrow \infty)$ and compare it to the similar expansion of a plane-wave in appropriate number of dimensions: $f_{\text {free }}^{3 D}(r) \propto 1-a_{3 D} / r, f_{\text {free }}^{2 D}(r) \propto \ln \left(r / a_{2 D}\right), f_{f r e e}^{1 D}(r) \propto r-a_{1 D}$. Expanding the solutions of Eq. (8) we find $f^{3 D}(r) \propto 1+(2 \gamma-1) / r-(\ln r) / r+\mathcal{O}\left(r^{-2}\right), f^{2 D}(r) \propto 2 \gamma-\ln r+\mathcal{O}\left(r^{-1}\right), f^{1 D}(r) \propto r+(2 \gamma-1)-\ln r+\mathcal{O}\left(r^{-1}\right)$, where $\gamma=0.577 \ldots$ is Euler's constant. There are logarithmic terms appearing in all dimensions. In a two dimensional system such a term is compatible with asymptotic behavior of a free-wave solution and it is possible to define a finite scattering length $a_{2 D}=e^{2 \gamma} r_{0}=3.172 \ldots r_{0}$. Indeed, numerical evaluation of the equation of state in a two-dimensional dipolar system [58] is in agreement with equation of state for hard-disks with same values of $a_{2 D}[59]$. Also, Bogoliubov theory at small $n a_{2 D}^{2}$ provides correct predictions for the correlation functions and condensate fraction. Thus, in two-dimensions dipolar $1 /|r|^{3}$ potential can be treated as a short-range one. Of course, values of the typical value of the gas parameter $n a_{2 D}^{2}$ for which universal in terms of $n a_{2 D}^{2}$ description starts being valid is smaller for dipolar interaction, than for "usual" short-range potentials (for example, a soft-disks [59]).

Instead, in a one-dimensional system the free-wave solution does not contain logarithmic term and this makes the dipolar solution be incompatible with it. For example, for short-range potentials $s$-wave scattering length can be calculated through the limit $\lim _{r \rightarrow \infty}\left[r-f(r) / f^{\prime}(r)\right]=a_{1 D}$. As it is easy to see, corresponding expression diverges in the case of dipolar interaction potential. It means that it is not possible to describe properties of a one-dimensional dipolar system by a short range potential. The same conclusion can be reached by comparing the ground-state energy of a repulsive dipolar system [27, 48] to ground-state energy of the repulsive short-range $\delta$-pseudpotential[30] in the dilute regime $n a_{1 D} \ll 1$. The leading term in the energy $E$ is the one of the Tonks-Girardeau gas $E_{T G} / N=\pi^{2} \hbar^{2} n_{1 D}^{2} / 6 M[26]$. The first correction should include (if the short-range description is possible) terms $n a_{1 D}$. Instead it is clear that it is not the case, as for Lieb-Liniger gas $a_{1 D}<0$ and energy is lowered $E<E_{T G}$. By simply neglecting the logarithmic term for dipoles one would find negative scattering length $a_{1 D}=(1-2 \gamma) r_{0}=-0.154 \ldots$, and the energy would be lower. But we definitely know that this is not the case, instead energy is higher $E>E_{T G}$. Thus we find problems trying to describe the dipolar interaction potential by a short-range model. Most probably, the same will happen in a three dimensional system.

\section{CONCLUSIONS}

In conclusions, we have studied possible signatures of a super-Tonks-Girardeau gas in a system of trapped quasi-onedimensional dipoles at zero temperature. This regime can be entered by exploiting a confinement induced resonance of the effective 1D scattering amplitude. Using previously calculated equation of state we provide predictions for the frequency of the lowest compressional mode. Properties in the high density regime are calculated within harmonic approximation. We provide an explicit mapping of the ground-state wave function of one-dimensional dipolar system of bosons; fermions and Bose-Fermi mixture and conclude that local properties and energy are the same at zero temperature. A question to which extent the dipolar potential can be treated long- or short- range is discussed. Superfluidity in one-dimensional systems is tested using different definitions.

During preparation of the paper to publication a related article [60] appeared, with a study of frequencies of collective oscillations. Their findings for the lowest breathing model are in agreement with ours. In addition frequencies of higher modes are calculated.

The work was partially supported by (Spain) Grant No. FIS2005-04181, Generalitat de Catalunya Grant No. 2005SGR-00779 and RFBR. G.E.A. acknowledges post doctoral fellowship by MEC (Spain).

\section{APPENDIX A: EQUATION OF STATE IN THE HIGH-DENSITY REGIME}

In the high density regime, $n r_{0} \gg 1$, potential energy dominates and properties of the system can be compared to the ones of a classical crystal with the lattice spacing $a$ defined by the density $a=n^{-1}$. Several terms of the expansion of the equation of state can be found using harmonic approximation (for $2 \mathrm{D}$ dipolar crystal this has been 


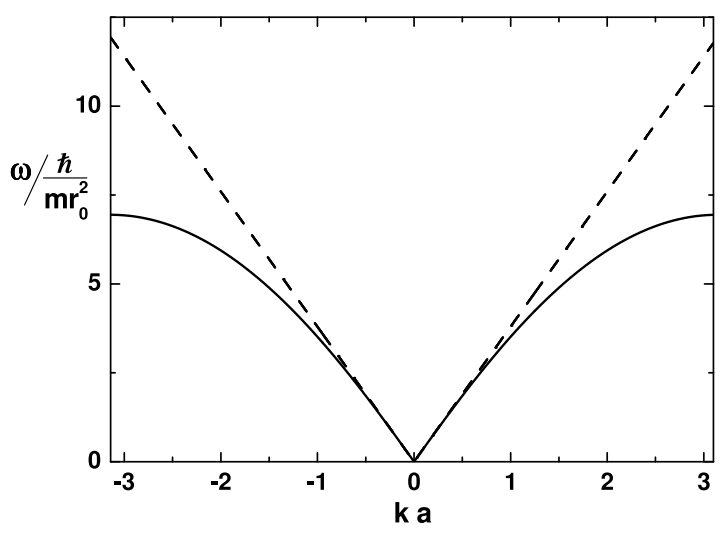

FIG. 2: Dispersion curve $\omega(k)$ in the first Brillouin zone $-\frac{\pi}{a}<k<\frac{\pi}{a}$ in the high density limit $n r_{0} \gg 1$. Solid line: harmonic approximation, Eq. (A2), dashed line: phonons, Eq. (A3).

done in Ref. [61]). The leading term is given by potential energy of a classical lattice

$$
\frac{E^{(0)}}{N}=\frac{\hbar^{2} r_{0}}{M} \sum_{j=1}^{\infty} \frac{1}{(j a)^{3}}=\zeta(3) \frac{\hbar^{2}}{M r_{0}^{2}}\left(n r_{0}\right)^{3}
$$

Particles move close to lattice sites $u_{j}=z_{j}-j a \ll a$. The potential energy can be expanded up to quadratic terms in $u_{j}$ and classical equation of motion can be solved looking for the wave solution $u_{j}=r_{0} e^{i(k j a-\omega t)}$. For the dipolar potential this leads to the following expression for the frequency $\omega$ :

$$
\omega^{2}(k)=\frac{12 \hbar^{2} r_{0}}{M^{2} a^{5}}\left(\sum_{j=1}^{\infty} \frac{2}{j^{5}}-\sum_{j=1}^{\infty} \frac{e^{j(i k a)}}{j^{5}}-\sum_{j=1}^{\infty} \frac{e^{j(-i k a)}}{j^{5}}\right)=\frac{12 \hbar^{2} r_{0}}{M^{2} a^{5}}\left(2 \zeta(5)-L i_{5}\left(e^{i k a}\right)-L i_{5}\left(e^{-i k a}\right)\right),
$$

where $\zeta(z)$ is the Riemann zeta function and $L i_{n}(z)$ is a polylogarithm function. The dispersion relation (A2) is shown in Fig. 2.

The low-momenta behavior $|k| \rightarrow 0$ can obtained by expanding exponents in the sums in $\mathrm{A2}$ and noticing that zeroand first- order terms get canceled

$$
\omega(k) \approx \sqrt{\frac{12 \hbar^{2} r_{0}}{M^{2} a^{5}} \sum_{j=1}^{\infty} \frac{k^{2} a^{2}}{j^{3}}}=2 \sqrt{3 \zeta(3)} \frac{\hbar}{M r_{0}}\left(n r_{0}\right)^{3 / 2}|k|
$$

Small $|k|$ behavior correspond to phonons. Same result can be recovered by calculating the compressibility in the system $m c^{2}=n \partial \mu / \partial n$, using the leading term in the energy (A1) to calculate the chemical potential $\mu=\partial E^{(0)} / \partial N$. Then the phononic spectra calculated as $\omega(k)=c|k|$ exactly coincides with expression (A3). In Fig. 2 the phonon excitation spectrum is plotted against the solution (A2). The latter solution has a characteristic rounding close to boundary of the Brillouin zone. This feature, of course, is missed in phononic description which becomes inapplicable for such large values of $|k|$.

Contribution $E^{(1)}$ to the lattice energy (A1) is obtained by summation of the energy of the zero-point motion of atoms. Thus, one has to integrate the dispersion (A2) over the first Brillouin zone (BZ):

$$
\frac{E^{(1)}}{N}=\int_{B Z} \frac{\hbar \omega(k)}{2} \frac{d k}{V_{B Z}}=C \frac{\hbar^{2}}{M r_{0}^{2}}\left(n r_{0}\right)^{5 / 2}
$$

The obtained contribution is positive, as it adds positive kinetic energy and describes displacement of atoms from the minimum of potential energy. Correction (A4) scales with density as $\left(n r_{0}\right)^{5 / 2}$ compared to the $\left(n r_{0}\right)^{3}$ dependence of the dominant term. The coefficient of proportionality $C=2.26 \ldots$ was obtained by numerical integration of (A4) with 
the dispersion relation (A2).

[1] A. Griesmaier, J. Werner, S. Hensler, J. Stuhler, and T. Pfau, Phys. Rev. Lett. 94, 160401 (2005).

[2] C. Haimberger, J. Kleinert, M. Bhattacharya, and N. P. Bigelow, Phys. Rev. A (2004).

[3] J. M. Sage, S. Sainis, T. Bergeman, and D. DeMille, Phys. Rev. Lett. 94, 203001 (2005).

[4] H. P. Büchler, E. Demler, M. Lukin, A. Micheli, N. Prokofev, G. Pupillo, and P. Zoller Phys. Rev. Lett. 98, 060404 (2007).

[5] D. Tong, S. M. Farooqi, J. Stanojevic, S. Krishnan, Y. P. Zhang, R. Côté, E. E. Eyler, and P. L. Gould, Phys. Rev. Lett. 93, 063001 (2004).

[6] M. D. Lukin, M. Fleischhauer, R. Cote, L. M. Duan, D. Jaksch, J. I. Cirac, and P. Zoller, Phys. Rev. Lett. 87, 037901 (2001).

[7] S. Giovanazzi, L. Santos, and T. Pfau, Phys. Rev. A 75, 015604 (2007).

[8] J. Stuhler, A. Griesmaier, T. Koch, M. Fattori, T. Pfau, S. Giovanazzi, P. Pedri, and L. Santos, Phys. Rev. Lett. 95, 150406 (2005).

[9] S. Yi and L. You, Phys. Rev. A 67, 045601 (2003).

[10] T. Lahaye, T. Koch, B. Frohlich, M. Fattori, J. Metz, S. G. A. Griesmaier, and T. Pfau, Nature 448, 672 (2007).

[11] R. Chicireanu, A. Pouderous, R. Barbé, B. Laburthe-Tolra, E. Maréchal, L. Vernac, J.-C. Keller, and O. Gorceix, Phys. Rev. A 73, 053406 (2006).

[12] J. M. Gerton, D. Strekalov, I. Prodan, and R. G. Hulet, Nature 408, 692 (2000).

[13] E. A. Donley, N. R. Claussen, S. L. Cornish, J. L. Roberts, E. A. Cornell, and C. E. Wieman, Nature 412, 0028 (2001).

[14] J. L. Roberts, N. R. Claussen, S. L. Cornish, E. A. Donley, E. A. Cornell, and C. E. Wieman, Phys. Rev. Lett. 86, 4211 (2001).

[15] L. Santos, G. V. Shlyapnikov, P. Zoller, and M. Lewenstein, Phys. Rev. Lett. 85, 1791 (2000).

[16] P. M. Lushnikov, Phys. Rev. A 66, 051601 (2002).

[17] S. Ronen, D. C. E. Bortolotti, D. Blume, and J. L. Bohn, Phys. Rev. A 74, 033611 (2006).

[18] U. R. Fischer, Phys. Rev. A 73, 031602 (2006).

[19] H. Moritz, T. Sẗoferle, M. Kohl, and T. Esslinger, Phys. Rev. Lett. 91, 250402 (2003).

[20] S. Richard, F. Gerbier, J. H. Thywissen, M. Hugbart, P. Bouyer, and A. Aspect, Phys. Rev. Lett. 91, 010405 (2003).

[21] I. Bloch, Nature Phys. 1, 23 (2005).

[22] P. C. Hohenberg, Phys. Rev. 158, 383 (1967).

[23] A. Altmeyer, S. Riedl, C. Kohstall, M. J. Wright, R. Geursen, M. Bartenstein, C. Chin, J. H. Denschlag, and R. Grimm, Physical Review Letters 98, 040401 (2007).

[24] G. E. Astrakharchik, J. Boronat, J. Casulleras, and S. Giorgini, Phys. Rev. Lett. 95, 190407 (2005).

[25] M. T. Batchelor, M. Bortz, X. W. Guan, and N. Oelkers, J. Stat. Mech. 2005, L10001 (2005).

[26] M. Girardeau, J. Math. Phys. (N.Y.) 1, 516 (1960).

[27] A. S. Arkhipov, G. E. Astrakharchik, A. V. Belikov, and Yu. E. Lozovik, JETP lett. 82, 39 (2005).

[28] B. L. Tolra, K. M. O'Hara, J. H. Huckans, W. D. Phillips, S. L. Rolston, and J. V. Porto, Phys. Rev. Lett. 92, 190401 (2004).

[29] T. Kinoshita, T. Wenger, and D. S. Weiss, Phys. Rev. Lett. 95, 190406 (2005).

[30] E. H. Lieb and W. Liniger, Phys. Rev. 130, 1605 (1963).

[31] G. E. Astrakharchik and S. Giorgini, Phys. Rev. A 68, 031602 (2003).

[32] G. E. Astrakharchik and S. Giorgini, J. Phys. B: At. Mol. Opt. Phys 39, S1 (2006).

[33] M. Olshanii, Phys. Rev. Lett. 81, 938 (1998).

[34] J. B. McGuire, J. Math. Phys (N.Y.) 5, 622 (1964).

[35] F. Mazzanti, G. E. Astrakharchik, J. Boronat, and J. Casulleras, arXiv:0705.4377 (2007).

[36] G. E. Astrakharchik, D. M. Gangardt, Y. E. Lozovik, and I. A. Sorokin, Phys. Rev. E 74, 021105 (2006).

[37] A. H. C. Neto, H. Q. Lin, Y. Chen, and J. M. P. Carmelo, Phys. Rev. B 50, 14032 (1994).

[38] G. E. Astrakharchik and L. P. Pitaevskii, Phys. Rev. A 70, 013608 (2004).

[39] M. A. Cazalilla, Journal of Physics B: AMOP 37, S1 (2004).

[40] Michael Pustilnik, Phys. Rev. Lett. 97, 036404 (2006)

[41] M. Khodas, M. Pustilnik, A. Kamenev, and L. I. Glazman, Phys. Rev. Lett. 99, 110405 (2007).

[42] E. L. Pollock and D. M. Ceperley, Phys. Rev. B 36, 8343 (1987).

[43] F. Calogero, J. Math. Phys. 10, 2191 (1969).

[44] B. Sutherland, J. Math. Phys 12, 246 (1971).

[45] L. S. Levitov and A. V.Shitov, Green's functions. Problems and solutions. 2nd edit. [in Russian] (Fizmatlit, 2002).

[46] E. H. Lieb, Phys. Rev. 130, 1616 (1963).

[47] M. Ishikawa and T. Takayama, J. Phys. Soc. Jpn 49, 1242 (1980).

[48] R. Citro, E. Orignac, S. D. Palo, and M. L. Chiofalo, Phys. Rev. A 75, 051602 (2007).

[49] G. E. Astrakharchik, Ph.D. thesis, Institute of Spectroscopy (Russian Academy of Sciences) (2005), URL http://isan.troitsk.ru/eng/eflns.htm.

[50] G. E. Astrakharchik, Phys. Rev. A 72, 063620 (2005). 
[51] C. Menotti and S. Stringari, Phys. Rev. A 66, 043610 (2002).

[52] G. E. Astrakharchik, D. Blume, S. Giorgini, and L. P. Pitaevskii, Phys. Rev. Lett. 93, 050402 (2004).

[53] M. Gaudin, Phys. Lett. 24A, 55 (1967).

[54] V. Y. Krivnov and A. A. Ovchinnikov, Sov. Phys. JETP 40, 781 (1975).

[55] C. N. Yang, Phys. Rev. Lett. 19, 1312 (1967).

[56] A. Imambekov and E. Demler, Phys. Rev. A 73, 021602 (2006).

[57] T. Dauxois, S. Ruffo, E. Arimondo, and M. Wilkens, eds., Dynamics and thermodynamics of systems with long-range interactions (Springer, Berlin, 2002).

[58] G. E. Astrakharchik, J. Boronat, J. Casulleras, I. L. Kurbakov, and Y. E. Lozovik, Phys. Rev. A 75, 063630 (2007).

[59] S. Pilati, J. Boronat, J. Casulleras, and S. Giorgini, Phys. Rev. A 71, 023605 (2005).

[60] P. Pedri, S. D. Palo, E. Orignac, R. Citro, and M. L. Chiofalo, arXiv:0708.2789 (2007).

[61] C. Mora, O. Parcollet, and X. Waintal, Phys. Rev. B 76, 064511 (2007). 